\title{
CRISES PARCIAIS MOTORAS INDUZIDAS POR MOVIMENTOS EM PACIENTES DIABÉTICOS
}

\author{
GLÓRIA M. A. S. TEDRUS * - MARIA CRISTINA ALBERTIN ** \\ NEWTON S. ODASHIMA * L LINEU C. FONSECA ***
}

\begin{abstract}
RESUMO - Levando em conta o número restrito de casos em âmbito internacional e a ausência de publicações na literatura nacional, os autores apresentam três casos de crises motoras focais induzidas por movimentos em pacientes diabéticos com mais de 50 anos de idade. As crises eram numerosas, sendo também desencadeadas ao serem assumidas determinadas posturas. Em dois casos havia hiperglicemia não cetótica. Todos os pacientes apresentavam hemiparesia e, em dois, tal déficit desapareceu após o cotnrole das crises. No outro paciente a hemiparesia era decorrente de acidente vascular cerebral isquêmico instalado 6 meses antes. Síndrome parietal transitória foi observada nos três pacientes. O EEG crítico mostrou, em dois pacientes, descargas na região parieto-occipital e, em um, na região temporal média contralateral. As crises foram resistentes aos anticonvulsivantes, só havendo controle com o tratamento dos distúrbios metabólicos. São discutidos aspectos clínico-eletrencefalográficos e etiopatogênicos. A pesquisa do caráter reflexo das crises e a dosagem da glicemia devem ser feitas em todos os pacientes idosos com crises motoras focais, face às implicações de ordem terapêutica e prognóstica.
\end{abstract}

Movement-induced seizures and diabetes mellitus

SUMMARY - Three elderly patients with partial motor seizures triggered by movement or posture of an extremity are presented. They had a history of diabetes mellitus. Two of them had nonketotic hyperglycemia. Hemiparesis was present in the three patients, which resolved in two. In the other patient, hemiparesis resulted from a previous stroke. All patients had transitory parietal syndrome. During seizures, BEG showed discharges in the parieto-occipital area in two cases and in the mid-temporal area in one. Seizures were resistant to conventional anticonvulsivante therapy, and ceased only after treatment of metabolic disturbances. A search for reflex seizures and hyperglycemia should be carried out routinely in the elderly with repeated spontaneous focal motor seizures. This may be important for treatment and prognosis.

A associação de distúrbios epilépticos, notadamente crises parciais motoras ou epilepsia contínua (crises motoras simples), e diabetes está bem documentada na literatura 2,5,14,17. Em alguns casos foi observada a indução dessas crises por movimentos 1.3,7,10,17. o reconhecimento dessa condição clínica é importante pelas implicações terapêuticas, pois essas crises são resistentes aos anticonvulsivantes, somente cedendo com a correção dos distúrbios metabólicos L 3 .

Levando em consideração, também, a raridade dos relatos na literatura, particularmente a inexistência em âmbito nacional, bem como algumas peculiaridades clínico-eletrencefalográficas críticas e intercríticas, apresentamos três casos de pacientes diabéticos com crises parciais motoras induzidas por movimentos.

Departamento de Neuropsiquiatria da Faculdade de Ciências Médicas da Pontifícia Universidade Católica de Campinas (PUCCAMP); * Professor Assistente; ** Neurologista do Hospital e Maternidade Celso Pierro, PUCCAMP; *** Professor Titular.

Dr. Lineu Corrêa Fonseca - Departamento de Neuropsiquiatria, Faculdade de Ciências Médicas, PUCCAMP - Av. John Boyd Dunlop s/n - 13060 Campimas SP - Brasil. 


\section{CASUÍSTICA}

Caso 1 - JB, paciente com 51 anos de idade, internado em 09/04/90 (Registro 00990010-1, HMCP-PUCCAMP) referindo há um mês episódios de formigamento no membro superior (MS) direito (D), de curta duração. Quinze dias após, tornaram-se mais numerosos e apresentavam a sequência de parestesias no MSD, movimentos clônicos desse membro e, finalmente, clonias do membro inferior (MI) D, sendo desencadeados por movimentos ou determinadas posturas. Apresentava história de hipertensão arterial leve, etilismo moderado e diabetes melito, fazendo uso de clorpropamida. Ao exame neurológico de admissão apresentava hemiparesia $D$, com (alteração da sensibilidade cinético-postural e astereognosia na mão D. Ao ser internado, a glicemia de jejum era $340 \mathrm{mg} / 100 \mathrm{ml}$, a osmolaridade plasmática 322,8 m0sm/L. e havia traços de corpos cetônicos na urina. O líquido cefalorraquidiano (LCR) inicial mostrava 9,3 leucócitos $/ \mathrm{mm} 3$ e glicose de $117 \mathrm{mg} / 100 \mathrm{ml}$. As radiografias de crânio foram normais. Descrição das crises e do EEG - As crises, com duração aproximada de três minutos, eram caracterizadas por clonias do MSD de pequena amplitude e frequência elevada, precedidas por parestesias desse segmento e seguidas, após intervalo de alguns segundos, por clonias de menor frequência do MID, principalmente do pé. Iniciavam-se durante os movimentos conjugados de abdução e elevação do MSD à altura da cabeça com flexão do antebraço sobre o braço ou segundos após, se mantida a postura. Durante as crises de movimentos clônicos do MSD registrou-se atividade rápida de pequena voltagem com pontas superpostas a cada $200-300 \mathrm{mseg}$ associadas a ondas lentas de pequena amplitude (Fig. 1). Essas descargas iniciavam-se nas regiões posteriores do hemisfério cerebral esquerdo (E) e difundiam-se, mas com predominio nítido nesse hemisfério. Já, acompanhando as clonias do MID, as descargas eram bilaterais sendo miaior o componente de ondas lentas. O EEG de repouso foi normal. Tratamento e evolução $-O$ paciente foi medicado com $1250 \mathrm{mg}$ de fenitoína endovenosa no lo dia, $300 \mathrm{mg}$ via oral nos dias subsequentes e com insulina NPH 80 (10 U) diariamente. Houve redução progressiva do número de crises que cessaram no $5^{\prime}$ dia de internação, com normalização do exame neurológico. Nessa ocasião a glicemia de jejum era $118 \mathrm{mg} / 100 \mathrm{ml}$.

Caso 2 - NMCP, paciente com 64 anos de idade, internada em 29/05/90 (Registro 00525052-S HMCP-PUCCAMP) referindo há 7 dias numerosas crises focais motoras no MSE, espontâneas ou desencadeadas por movimentos e por estímulos tateis na mão E. Apresentava história de hipertensão arterial leve e diabetes melito, fazendo uso de clorpropamida. Na admissão apresentava hemiparesia $E$, alteração da sensibilidade cinético-postural no MSE, hipopalestesia em membros interiores e hipoestesia superficial nas extremidades de membros inferiores e superiores. Mostrava também astereognosia no MSE. Ao ser internada, a glicemia de jejum era $340 \mathrm{mg} / 100 \mathrm{ml}$ e a osmolaridade plasmática $327,2 \mathrm{m0sm} / \mathrm{L}$. O exame de urina evidenciava traços de corpos cetônicos. No LCR a glicose era de $170 \mathrm{mg} / 100 \mathrm{~mL}$ Descrição das crises e do EEG - As crises eram caracterizadas por movimentos clônicos em flexão dos dedos da mão E, de pronação do antebraço $E$ ou flexão plantar do pé E. Frequentemente eram desencadeadas por movimentos de fechar a mão $E$ ou elevar o MSE. O EEG apresentava surtos com duração variável, de segundos a vários minutos, de ondas lentas rítmicas por vezes com pequenas pontas associadas, com nitido predomínio no hemisfério cerebral $D$, particularmente na região temporal média (Fig. 2). Essas descargas podiam ser associadas ou não a crises clônicas (espontâneas ou induzidas por movimentos). Por vezes as manifestações clínicas criticas não eram acompanhadas de modificações do traçado. A atividade elétrica cerebral de repouso era desorganizada na região fronto-temporal $D$, com melhora progressiva após o controle das crises. Tratamento e evolução - Foi feita, inicialmente, correção dos distúrbios hidroeletróliticos e administrada insulina simples. Mesmo com a associação subsequente de glibencLamida os níveis de glicemia mantiveram-se elevados, só havendo redução com a introdução de insulina NPH, no 9? dia de internação. Houve progressiva redução do número de crises, que cessaram no $4^{*}$ dia, embora persistisse glicemia entre 200 e $350 \mathrm{mg} / 100 \mathrm{ml}$ e traços de corpos cetônicos na urina. Não foram administrados anticonvulsivantes.

Caso 3 - PP, paciente com 62 anos de idade, internado em 21/03/90 (Registro 0080005-0 HMCP-PUCCAMP) referindo há 24 horas crises frequentes de movimentos clônicos no MSE, espontâneas ou induzidas por movimentos. Apresentava história de duas internações (20/01/85 e 22/08/89) com episódios de hemiparesia $E$ transitória e crises possivelmente tônico-òlônicas generalizadas. Em outubro-1989 houve instalação de provável acidente vascular cerebral, com hemiparesia $\mathbf{E}$ e imagem hipodensa frontal e temporal $D$ à tomografia computadorizada de crânio (TC). Desde março-1988 tem diagnóstico de diabetes melito controlado somente com dieta hipocalórica. Era hipertenso arterial leve e etilista moderado. O exame neurológico, na admissão, revelava hemiparesia $E$ com sinal de Babinski e hemianopsia homônima $E$. 

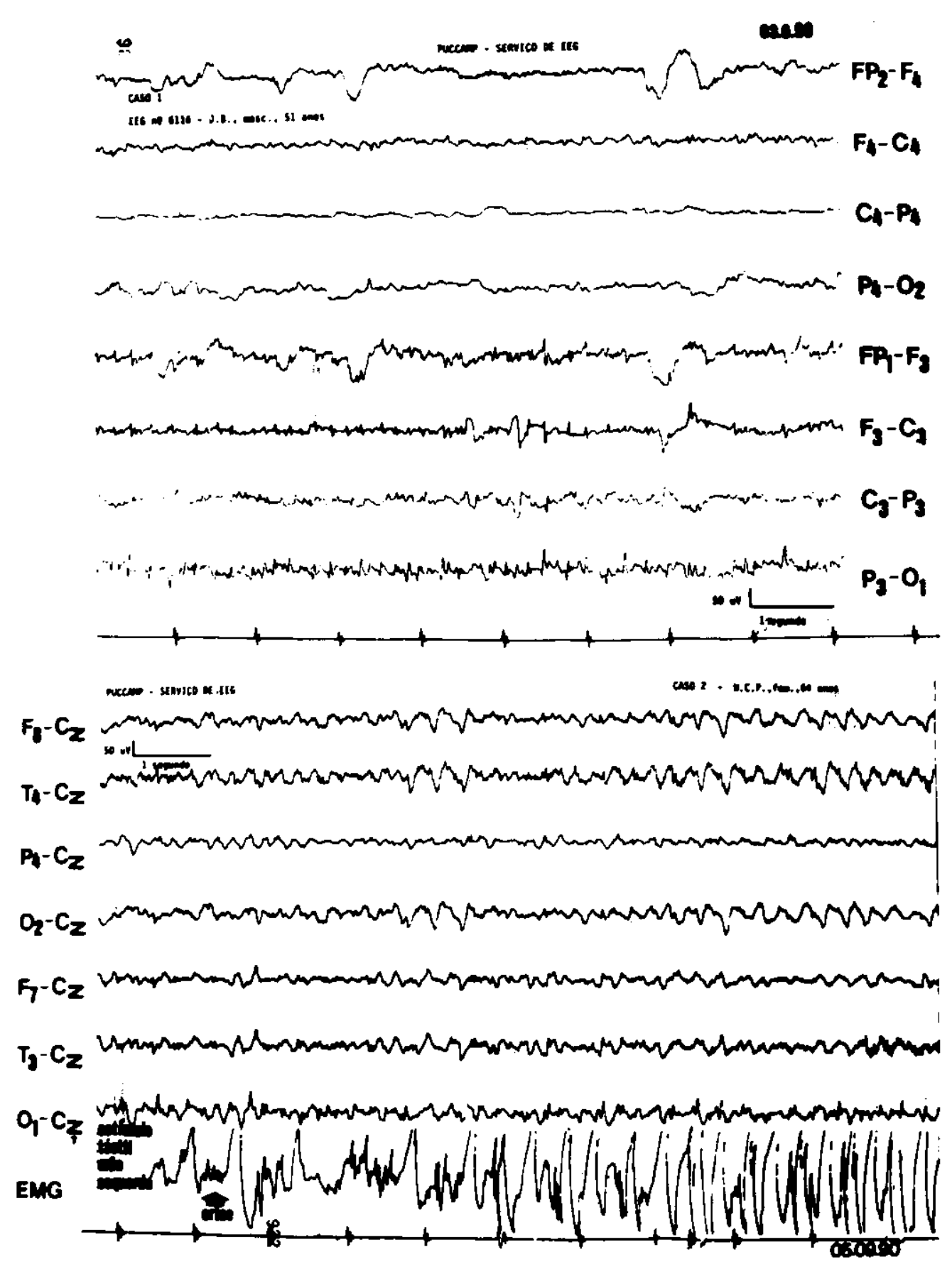

$\mathrm{C}_{4}-\mathrm{C}_{4}$

T.-P.

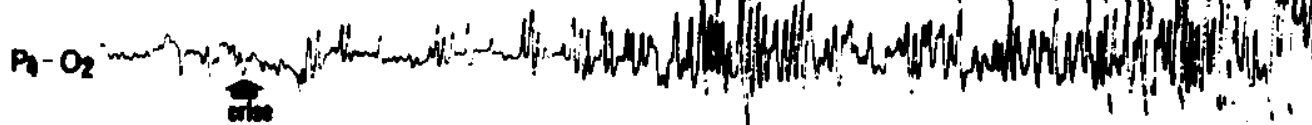

$F_{2} \cdot c_{3}$

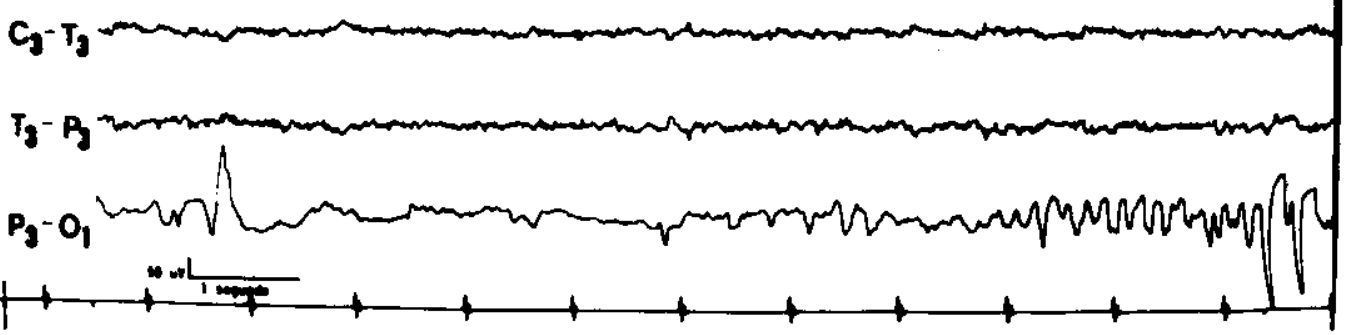

Fig. 1 (no alto) - Caso 1. EEG durante clonias do membro superior direito induzidas por movimentos. Atividade ropida com pontas $e$ ondas lentas associadas, no hemisferio esquerdo.

Fig. \& (no meio) - Caso \&. EEG durante crise clonica da mão esquerda desencadeada por estimulaço tatil. Ondas ritmicas por vezes com pequenas pontas associadas, predominando no hemisforio direito.

Fig. 3 (em baixo) - Caso 3. EEG ictal. Descargas de pontas ritmicas a 11-12 Hz de amplitude crescente na região parieto-occipitial direita. 
Apresentava, também no MSE, alteração da sensibilidade cinético-postural, astereognosia e discriminação de dois pontos comprometida. A glicemia de jejum esteve discretamente aumentada apenas em curto período de tempo durante a 2 a semana de internação. A osmo1 aridade plasmática na internação foi $307 \mathrm{mOsm} / \mathrm{L}$. O LCR suboecipital e as radiografias de crânio foram normais. Descrição das crises e do EEG - Nas crises ocorriam leve flexão do antebraço sobre o braço, abdução do membro superior E, movimentos clônicos em flexão do antebraço, mãos e dedos e, por vezes, contraçõs clônicas da musculatura torácica à E. As crises eram desencadeadas por vários tipos de movimentos do MSE, como pegar objetos com a mão ou ao «assumir a postura de elevação acima da cabeça. Durante as crises registravamse na região parieto-occipital $D$ descargas de pontas rítmicas a 11-12 Hz de amplitude progressivamente maior e, depois de alguns segundos, com ondas lentas intercaladas compondo complexos irregulares a $5 \mathrm{~Hz}$ e eventual propagação para região occipital E (Fig. 3). As descargas duravam aproximadamente 40 segundos. Nas crises de menor duração (10-20 segundos), registravam-se na região parieto-occipital $D$ descargas rítmicas a $7-12$ Hz de amplitude crescente. Essas descargas ocorriam por vezes durante a movimentação do MSE, sem o desencadear de crise clínica. No traçado de repouso apareciam ondas agudas na região occipital $D$ e, em menor número, de modo independente, na região temporal média $D$, assim como numerosos grupos de ondas lentas na região fronto-temporal D. Tratamento e evolução - Apesar de ter sido administrada DPH endovenosa $1000 \mathrm{mg}$ no $V>$ dia de internação, $250 \mathrm{mg}$ endovenoso \& $300 \mathrm{mg}$ via oral no 2 ? dia e $300 \mathrm{mg}$ via oral nos dias subsequentes, apenas houve redução do número de crises a partir do $4 *$ dia e controle total no $7 *>$ dia.

\section{COMENTARIOS}

Crises focais e epilepsia parcial contínua foram relatadas nas fases iniciais dt hiperglicemia não cetótica em pacientes diabéticos não insulinodependentes 2,5,14,17. Em alguns desses casos, geralmente pessoas com idade superior a 50 anos, as clonias focais eram induzidas por movimentos ativos ou determinadas posturas do segmento envolvido na crise 1.3,10,17. $p_{0} r$ outro lado, crises epilépticas induzidas por movimentos podem ocorrer em jovens não diabéticos, mas são tônicas e desencadeadas por movimentos bruscos ou sobressalto 4,6,9,12,13,18.

Em pacientes diabéticos existiriam vários fatores implicados na etiopatogenia das crises, como alterações metabólicas que ocorrem nas fases iniciais da hiperglicemia não cetótica e as possíveis lesões estruturais pré-existentes 1,3,10,17. Em alguns casos, principalmente na epilepsia parcial contínua, existe lesão orgânica cerebral definida, mas o exato mecanismo que desencadeia as crises continua não totalmente esclarecido. Foi sugerido por vários autores $2,3,10,15,16,17$ q e uma lesão cerebral aguda ou cicatricial, possivelmente de etiologia vascular, tenha importante papel na gênese das crises. O diabetes frequentemente leva a complicações cerebrais pela microangiopatia com pequenas áreas isquémicas ou com suplencia diminuída e essas áreas poderiam ser sensíveis aos distúrbios metabólicos.

As alterações metabólicas atuariam como um conjunto de mecanismos que levaria a diminuição do limiar convulsiógeno e maior excitabilidade neuronal nessas áreas lesadas. Isso ocorreria devido a alcalose intracelular, com inibição do ciclo de Krebs e consequente diminuição dos níveis de GABA. Associa-se, também, o fato de que a hiperglicemia e a consequente hemoconcentração levam a diminuição do fluxo sanguíneo cerebral e desidratação neuronal. Corroborando a importância do fator metabólico, na literatura é mostrado que as crises são usualmente refratárias ao uso de anticonvulsivantes. Isto ocorreu no caso 1 , onde só houve o controle total das crises após a administração de insulina, reposição de eletrólitos e hidratação, não tendo cedido com o uso inicial de anticonvulsivantes. Já no caso 2 , em que não foi utilizada medicação antiepiléptica, as crises cessaram ainda na vigência de hiperglicemia, sugerindo que outros distúrbios estivessem envolvidos.

No caso 3, embora o paciente fosse diabético, não ficou evidente a correlação entre hiperglicemia e crises. No entanto, a medicação anticonvulsivante utilizada não levou ao controle imediato das crises. Esse paciente apresentava sequela de acidente vascular cerebral isquémico, precedendo o quadro atual e com lesão à TC. É possível que, neste caso, alterações metabólicas de pequena monta tenham podido desencadear as crises devido à lesão cerebral prévia.

Em todos os nossos pacientes ocorreram déficits sensitivos e motores nos segmentos envolvidos nas crises, que regrediram após o seu controle de modo semelhante 
ao assinalado na literatura e sugerindo que os deficits sejam de natureza pós-ictal. Em nossos três casos o exame neurológico revelava síndrome parietal e, em dois deles, o EEG mostrava descargas críticas na região parietal e occipital. Essas alterações clínico-eletrencefalográficas desapareceram após o controle das crises, sugerindo participação importante do lobo parietal na gênese dessas crises.

Nossos dados, aliados aos da literatura, mostram que a identificação de crises motoras induzidas por movimentos é de grande importância no indicar distúrbios metabólicos ligados ao diabetes. O diagnóstico adequado dessa condição clínica é fundamental, pois o controle das crises só será obtido com a correção dos distúrbios metabólicos. A investigação do caráter reflexo das crises e de distúrbios metabólicos do diabetes deve ser rotina em pacientes com crises motoras focais repetidas.

\section{REFERÊNCIAS}

1. Aquino A, Babos AJ. Movement-induced seizures in nonketotic hyperglycemia. Neurology 19830, 3000:600-604.

2. Askenasy JH, Strieifler M, Carasso R. Moderate nonketotic hyperglycemia: a cause of focal eqpiilkepssyy.. Eluu $r \mathrm{~N}$ \&enob119[97,7,1616:51-61.

3. Brick JP, Gutrecht JA, Ringel RA. Reflex epilepsy and nonketotic hyperglycemia in

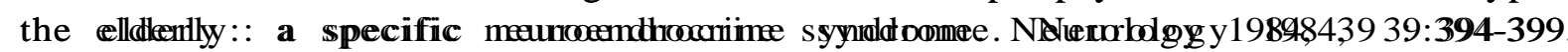

4. Buscaino GA, Striano S, Meo R, Bilo L. Reflex epilepsy: a proposal for classification

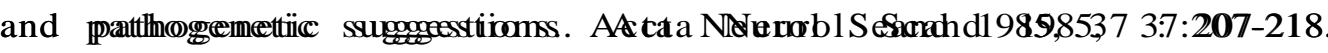

5. Daniels Jd. Chokroverty S, Barron KO. Anacidotic hyperglycemia and focal seizures Arch Intteerm Miseld 1969,9,12424:701-706

6. Falconer MA. Driver MV, Serafenitides EA. Seizures induced by movement: report of

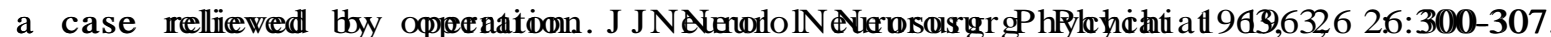

7. Gabor AC. Focal seizures induced by movement without sensory feedback mechanisms. Electroenceph Clin Neurophysiol 1974, 36:403-408.

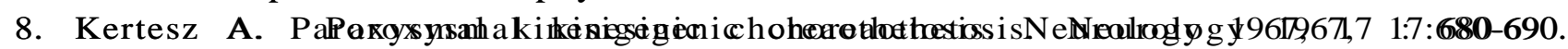

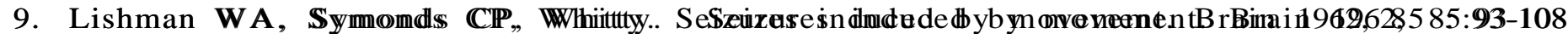

10. Maccario M, Brooblyn NY. Neurological dysfunction associated with nonketotic hyperglycemia. Ancedh Meeurod1988,8,1919:535-531.

11. Maccario M. Messis CP. Vastola EF. Focal seizures as a manifestation of hyperglyce-

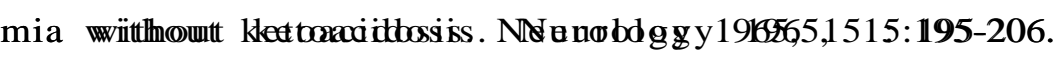

12. Perez-Borja C. Tassinari AC. Swanson AG. Paroxysmal choreoathetosis and seizures

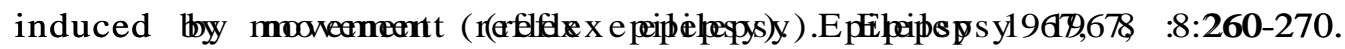

13. Pitha V - Eppilleppsiie reffeexee. RR e vNAwrorbl19B8387070:1178-181.

14. Singh BM, Gupta D. Strobos RJ. Nonketotic hyperglycemia and epilepsia partialis continua. Arrodh Neeurod1 19983.32929:187-190.

15. Sing BM, Strobos RJ. Epilepsia partialis continua associated with nonketotic hyperglycemia: clinical and biochemical profile of 21 patients. Ann Neurol 1980, 8:155-160.

16. Vastola EF. Maccaro M. Homan R. Activation of epileptogenic foci by hyperosmolality. Neurology 19867.177:520-526.

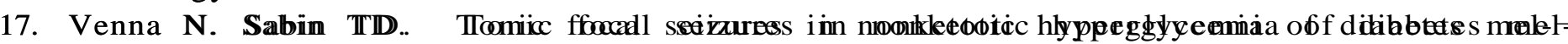
litus. Anrodh Neaurod1988,1,3838:512-514.

18. Whitty CWM. Lishman WA. Fitz Gibbison JP. Seizures induced by movement: a form of meeffleex eppileppssy. Lhacetet196964,1 1:1403-1406. 